\title{
Vesicle adhesion visualized with total internal reflection imaging ellipsometry biosensor
}

\author{
Li Liu $^{\mathrm{a}}$, Annie Viallat ${ }^{\mathrm{b}, *}$, Gang Jin ${ }^{\mathrm{a}}$ \\ a Institute of Mechanics, Chinese Academy of Sciences, 100190 Beijing, China \\ b Aix Marseille Université, CNRS, LAI, UMR 7333, Inserm UMR 1067, 13009, Marseille, France
}

\section{A R T I C L E I N F O}

\section{Article history:}

Received 8 May 2013

Received in revised form 2 August 2013

Accepted 11 August 2013

Available online 31 August 2013

\section{Keywords:}

Giant lipid vesicles (GUVs)

Vesicles adhesion

Non-specific adhesion

Total internal reflection imaging

ellipsometry (TIRIE)

Biosensor

\begin{abstract}
A B S T R A C T
The behavior of giant lipid vesicles (GUVs) interacting with an adhesive surface coated with poly-L-lysine is investigated by a biosensor based on total internal reflection imaging ellipsometry (TIRIE). The adhesion of GUVs on the adhesive surface is regulated by the concentration of the poly-L-lysine coating, the $\mathrm{pH}$ of the vesicle suspension, and the flow rate of the suspending fluid. The TIRIE biosensor detects very clearly and sensitively GUV adsorption, flattening, rupture, and de-adhesion, all these behaviors being independently observed by phase contrast microscopy. These results show that the setting of the TIRIE biosensor, optimized for molecular sensing can be extended to GUV sensing. This work paves the way to develop a new sensitive high-throughput, low-consumption and low-cost biosensor technology for micron-size objects such as cells, capsules and liposomes.
\end{abstract}

(C) 2013 Elsevier B.V. All rights reserved.

\section{Introduction}

Worldwide, cell biosensors are the focus of considerable and increasing interest in biomedical research, environmental detection, etc. [1] since individual cells are increasingly regarded as the basic unit of our understanding of biology and are often key markers for clinical applications. By comparison with conventional methods [2] such as chromatographic, spectroscopic, atomic absorption and immunoassay techniques used for cell detection and characterization of their behavior, cell biosensors are accurate and sensitive low cost devices. They are easy to implement and provide fast responses [3]. Label-free and non-invasive biosensors such as acoustic [4], electrical [5] and optical biosensors offer generic, integrated and dynamic information on cell adhesion. Label-free optical biosensors are increasingly used to study cell adhesion, spreading and de-adhesion at surfaces partly because of their fast response, their absence of electromagnetic interference and their high sensitivity [6]. So far, they are based on the propagation of a surface-bound electromagnetic wave, which is achieved on thin metal films using light excited surface plasmon resonance (SPR) [7] or on dielectric substrates using diffraction grating coupled waveguide mode resonances (RWG) [8,9]. Like SPR and RWG, optical biosensors based on ellipsometry [10] have the advantage to provide time and

\footnotetext{
* Corresponding author.

E-mail addresses: annie.viallat@inserm.fr (A. Viallat), gajin@imech.ac.cn (G. Jin).
}

spatial resolution when conventional photodetectors are replaced with CCD cameras. Optical imaging ellipsometry offers real-time monitoring of binding events with a lateral resolution limited to a couple of micrometers. It is used to probe biomolecular interactions at solid-liquid interfaces and has demonstrated its capacity to characterize DNA and protein microarrays, to detect tumor and hepatitis markers, antibody screening or virus recognition [11], or to monitor lipid phase separation in a supported lipid bilayer [12]. Total internal reflection imaging ellipsometry (TIRIE) was found to be more sensitive than other reflectivity related methods in measuring the interaction between bio-molecules [13]. Very recently, surface-enhanced ellipsometric contrast (SEEC) imaging [14], which uses substrates made of specifically designed multiple dielectric layers to enhance the contrast by a factor 100 in comparison with conventional bright field, successfully resolved binding kinetics of an antibody to phase-separated lipid bilayers [15]. However, up to now, optical ellipsometry biosensors have focused on bio-molecular recognition by their specific binding to molecules located on the substrate (either immobilized or embedded in a solid supported lipid bilayer) and not on adhesion or spreading of objects of size of about ten microns as cells.

The aim of this paper is to show that TIRIE biosensor can be used for cell sensing to detect and measure adhesive states of cells and micron-size capsules. This biosensor is developed for over ten years [16]. It has been thoroughly described in Ref. [17] and is illustrated in Fig. 1. It is based on imaging ellipsometry performed in a total internal reflection mode combined with a 24 independent 


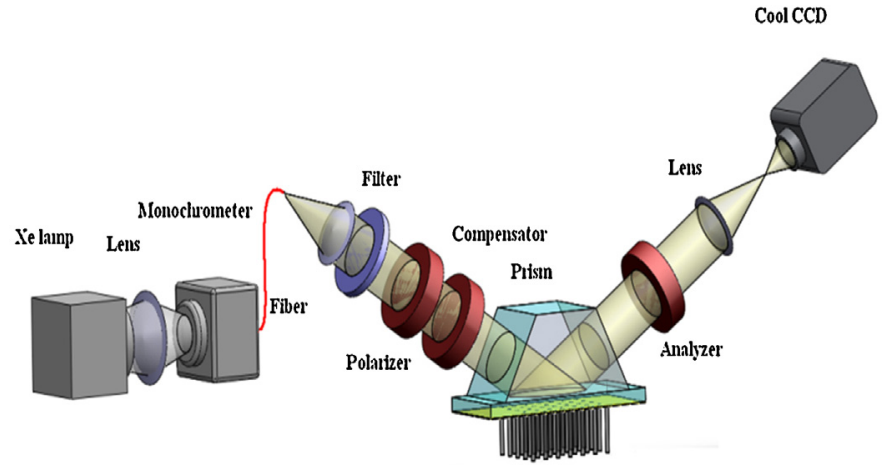

Fig. 1. Schematic of the TIRIE biosensor.

channel microfluidic reactor array to implement real-time microarray visualization for high throughput analysis.

In TIRIE biosensor, the probe light is an evanescent wave created on the gold surface, which propagates vertically in the sample solution within a very limited thickness of a few hundreds nanometers. The bottom part of vesicles, located in the propagation zone of the evanescent wave, is detected by the probe.

The data acquisition used for the biosensor sampling is the imaging ellipsometry performed in the mode of Null and Off-null ellipsometry. The biosensor signal is the intensity change detected on the CCD camera, due to the change of polarization of the reflected light beam on the substrate modified by the presence of adhered vesicles. The pixel size in the image of the detected sample is larger than the size of single vesicles, so the intensity change per pixel is an average signal. The total signal is proportional to the amount of vesicles adhered on the substrate.It is noteworthy that in the biosensor detection, the knowledge of the absolute values of the refractive index and of the thickness of the lipid bilayer of the vesicles is not necessary.

We propose a first case study, based on the non-specific adhesive interaction of giant lipid vesicles (GUVs) [18] with a substrate coated with a charged polymer, namely poly-L-lysine. The adhesion between a GUV and a surface has been extensively studied in the last twenty years [19-23]. An easy way to modulate the adhesive interaction between a lipid bilayer and a substrate is to use non-specific electrostatic interaction. Zwitterionic lipids interact with positively charged polymers coated on the substrate. Here the substrates are coated by incubation of a solution of positively charged poly-L-lysine. In this work, vesicle adhesion and de-adhesion phenomena are visualized by TIRIE biosensor and controlled by phase contrast microscopy. We manage to detect various states presented by adhesive GUVs: pure settling, weak adhesion and de-adhesion, strong adhesion and membrane rupture.

\section{Experiments}

\subsection{Materials and methods}

All products are purchased from Sigma except 1-(3dimethylaminopropyl)-3-ethylcarbodimide hydrochloride (EDC) and $\mathrm{N}$-hydroxy-droxysuccinimid-e (NHS), which are purchased from ACROS. 1,2-dioleoyl-sn-glycero-3-phosphocholine (DOPC) vesicles are prepared by the standard electro formation method at $10 \mathrm{~Hz}$ and $1.5 \mathrm{~V}[24,25]$. They are filled with a sucrose solution of concentration $0.315 \mathrm{~mol} / \mathrm{L}$ and are suspended in a glucose solution of concentration $0.315 \mathrm{~mol} / \mathrm{L}$ to ensure iso-osmolarity in the vesicles and the suspension.

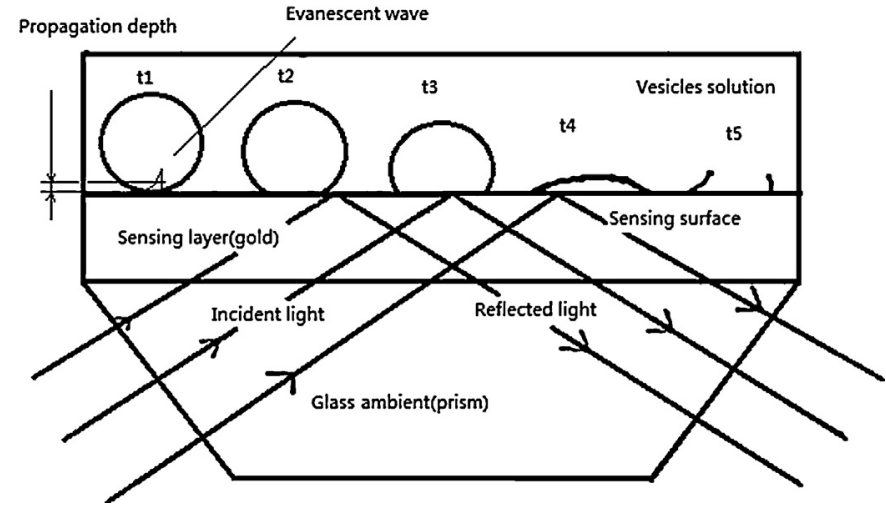

Fig. 2. Vesicles after sedimentation in a channel of the micro-fluidic reactor on the TIRIE biosensor. Possible shapes: $\left(t_{1}\right)$ spherical shape, no adhesion; $\left(t_{2}\right)$ truncated shape, weak adhesion; $\left(t_{3}\right)$ hemisphere, strong adhesion; $\left(t_{4}\right)$ aggregates of lipids after vesicle burst; $\left(t_{5}\right)$ some small fragments. The probe light beam from the light source transmits into the glass ambient (prism), penetrates the interface of the ambient and the sensing layer, and then transmits into the sensing layer until it reflects from the sensing surface. An evanescent wave appears at the sensing surface and propagates into the vesicle solution whereas the incident light reflects from the sensing surface. The shape modification on the sensing surface can be detected by the evanescent wave. The interaction between vesicles and the sensing surface is detected by the reflected light.

\subsection{TIRIE biosensor}

The TIRIE biosensor uses an expanded light beam at $633 \mathrm{~nm}$ wavelength as a light source and images with a CCD camera, pixel size of $13 \times 13 \mu \mathrm{m}^{2}$. The incident light beam goes through the polarizer, the compensator and the $59^{\circ}$ prism where the evanescent wave is used as optical probe to detect the bottom of the vesicles adhered onto the substrate. The incident light beam is reflected at the interface between the substrate and the tested solution and then passes through an analyzer and is focused on a sensing area of the charge coupled device (CCD) camera. The polarization axis of the light reflected in absence of vesicles is set in the Null ellipsometry condition (perpendicular to the analyzer corresponding to an absolute minimum of light flux detected at the CCD camera). The biosensor signal is the intensity change detected on the CCD camera, due to the change of polarization of the reflected light beam induced by the presence of vesicles adhered on the substrate. The image signal is recorded in 16 bit $(0-65,536)$ grayscale format. As a sensing surface, a gold layer of $30 \mathrm{~nm}$ thick on a glass slice optically coupled with a glass prism touches the tested solution. Each sensing micro-channel of the micro-fluidic reactor array system visualized by the TIRIE biosensor is about $1 \times 3 \mathrm{~mm}^{2}$ in area and $150 \mu \mathrm{m}$ in height. The flow into the micro-channels is driven by a peristaltic pump with $3 \times 8$ channels (ISMATEC) [26]. Vesicles are slowly transported to the sensing micro-channels of the microfluidic reactor array system. A sensing micro-channel is a chamber composed of Polydimethylsiloxane (PDMS) and a gold surface covered by a poly-L-lysine layer where vesicles are let to sediment and interact with the substrate (see Fig. 2). The vesicle behavior is analyzed in real-time.

\subsection{Optical microscopy}

Small observation chambers are made to observe the behavior of vesicles on the gold surface covered with poly-L-lysine. The same substrates used for TIRIE experiments are used for microscopy experiments. Vesicles are gently injected into the observation chamber, let to sediment for $5 \mathrm{~min}$ and then observed by phase contrast microscopy. Pictures are taken with an Andor camera using 

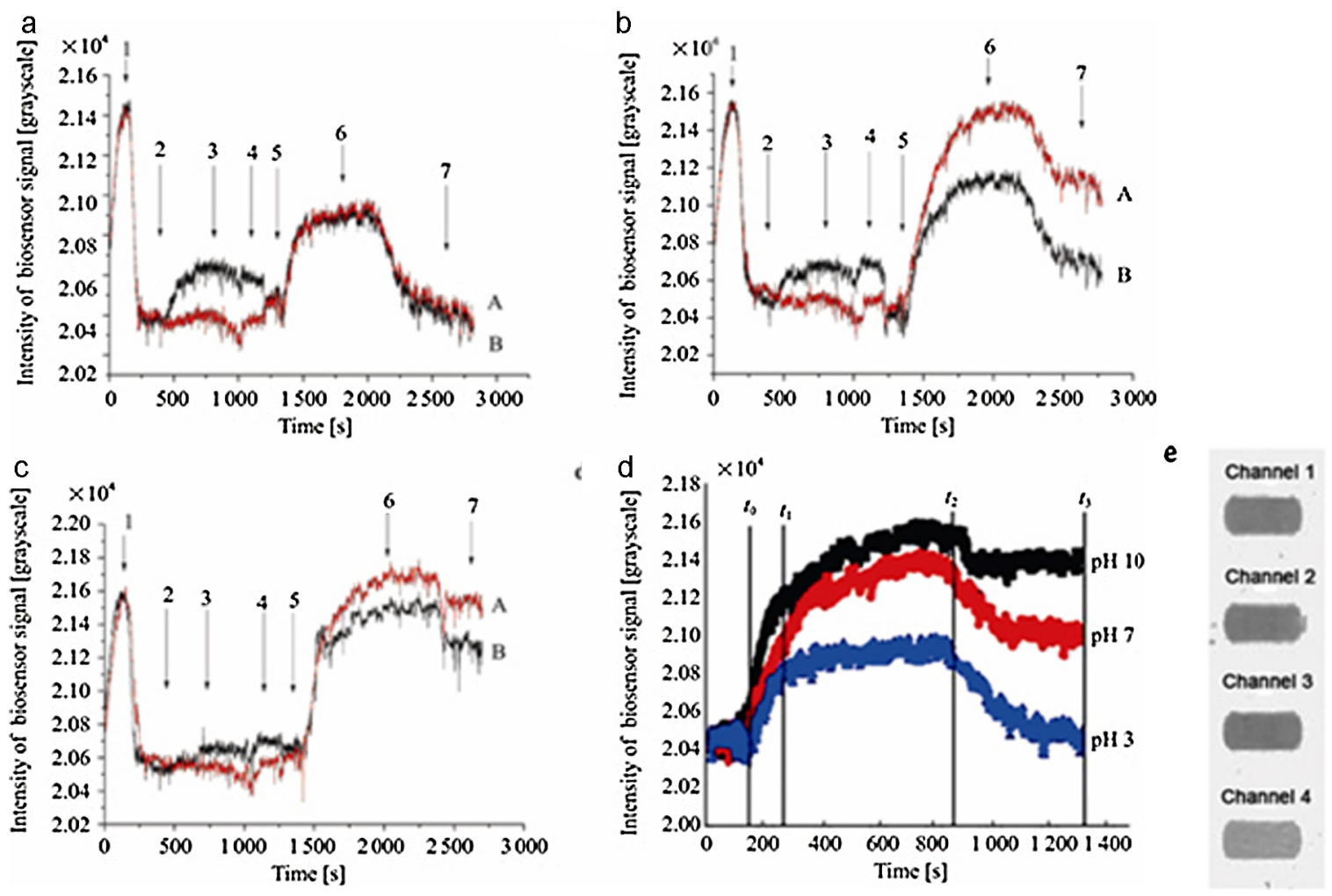

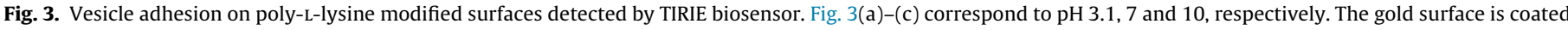

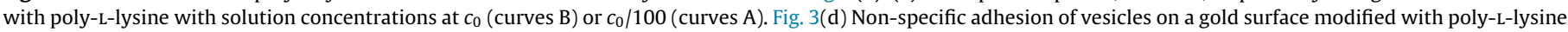

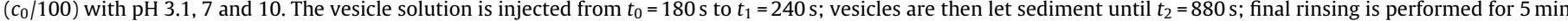

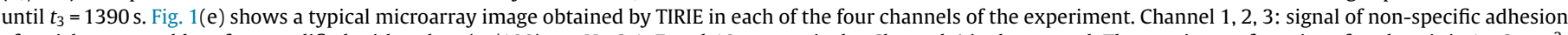

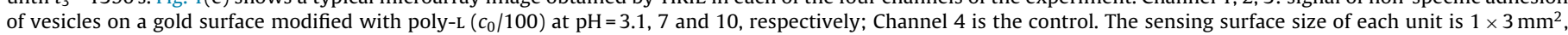
which is imaged in $13 \times 13$ pixels.

a phase contrast objective $\mathrm{x} 20$ on an ECLIPSE Ti-U Nikon inverted Microscope.

\subsection{Experimental procedure}

A solution of NHS and EDC is injected into the channel to activate the gold surface (Step 1 in Fig. 3(a)-(c)). After extensive rinsing with water, the poly-L-lysine solution (concentration $c_{0}=1 \mathrm{mg} / \mathrm{mL}$ or 1:100 dilution $c_{0} / 100$ ) is injected into the channels and let to incubate for 5 min (Step 3 in Fig. 3(a)-(c)). After water rinsing, a layer of poly-L-lysine is adsorbed onto the gold surface. As clearly seen in Fig. 3(a)-(c) step 3, the intensity of the TIRIE biosensor signal of the poly-L-lysine adsorption at concentration $c_{0}$ is higher than that in the case of adsorption at $c_{0} / 100$. Then a glucose solution (concentration of $0.3 \mathrm{~mol} / \mathrm{L}$ and $\mathrm{pH}$ at $3.1,7$ or 10 ) is injected into the channel to calibrate the TIRIE signal before the vesicles are introduced (Step 5 in Fig. 3(a)-(c)). The vesicle suspension solution (initial vesicle suspension diluted 5 times in glucose solution) is then injected at a flow rate of $5 \mu \mathrm{L} / \mathrm{min}$ for $600 \mathrm{~s}$ (Step 6 in Fig. 3(a)-(c)). The vesicles diluted in glucose solution slowly settle on the bottom of the micro-channel since they are filled with a sucrose solution, which is denser than the glucose solution. Then they would adhere on the substrate. Rinsing with glucose solution is subsequently performed.

\subsection{Contribution to the TIRIE signal induced by the adhesion of a vesicle}

The size of the vesicles is much larger than the propagation depth of the evanescent wave. The TIRIE signal due to the adhesion of a vesicle therefore results from the propagation of the evanescent wave first across the lipid bilayer and then within the internal glucose solution of the vesicle. All adhering vesicles of similar size contribute identically to the TIRIE signal since all vesicles have identical lipid membrane and identical internal solution. The average size of a vesicle is about $10 \mu \mathrm{m}$ and is smaller than the pixel size $\left(13 \times 13 \mu \mathrm{m}^{2}\right)$ of image by CCD camera. The detail of the adhesion area of a vesicle is therefore not imaged by the camera, which detects an average intensity of the vesicle image. Single vesicle adhesion is detected in average by a change of the intensity of a pixel of the camera. The light intensity received by the camera increases linearly with the number of vesicles adhered on the substrate.

\section{Results}

We use poly-L-lysine coating on the surface of the substrate to create and modulate adhesive interactions between the surface of the substrate and the vesicles. Poly-L-lysine is a linear polypeptide. It is a weak polyelectrolyte (only partially charged at moderate $\mathrm{pH}$ near its $\mathrm{pK}$ of 9.36), which interacts with lipids through $\mathrm{NH}^{+}$ groups. The $\mathrm{pH}$ and the ionic strength environments greatly influence poly-L-lysine dissociation, and, consequently the electrostatic interaction between the lipid membrane of the vesicles and the coated substrate [27]. Two concentrations of poly-L-lysine solutions are used to coat the gold surface of the substrates, $c_{0}=1 \mathrm{mg} / \mathrm{ml}$ and 1:100 dilution $c_{0} / 100$, referred hereafter as $c_{0}$ and $c_{0} / 100$, respectively. The vesicle adhesion is studied under three different $\mathrm{pH}$ conditions of the suspending glucose solution, $\mathrm{pH}=3.1,7$ and 10 . When the $\mathrm{pH}$ increases, the degree of dissociation of the surface functional groups of poly-L-lysine decreases and the interaction with the lipid membrane of vesicles weakens. This allows to 
Table 1

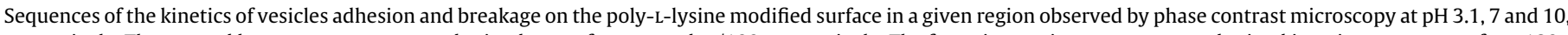

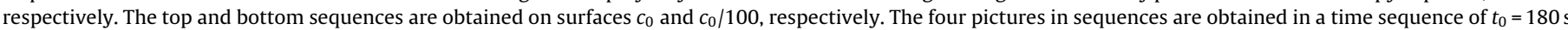

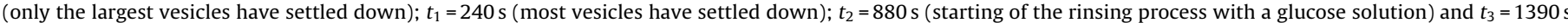
(end of the $5 \mathrm{~min}$ rinsing stage).

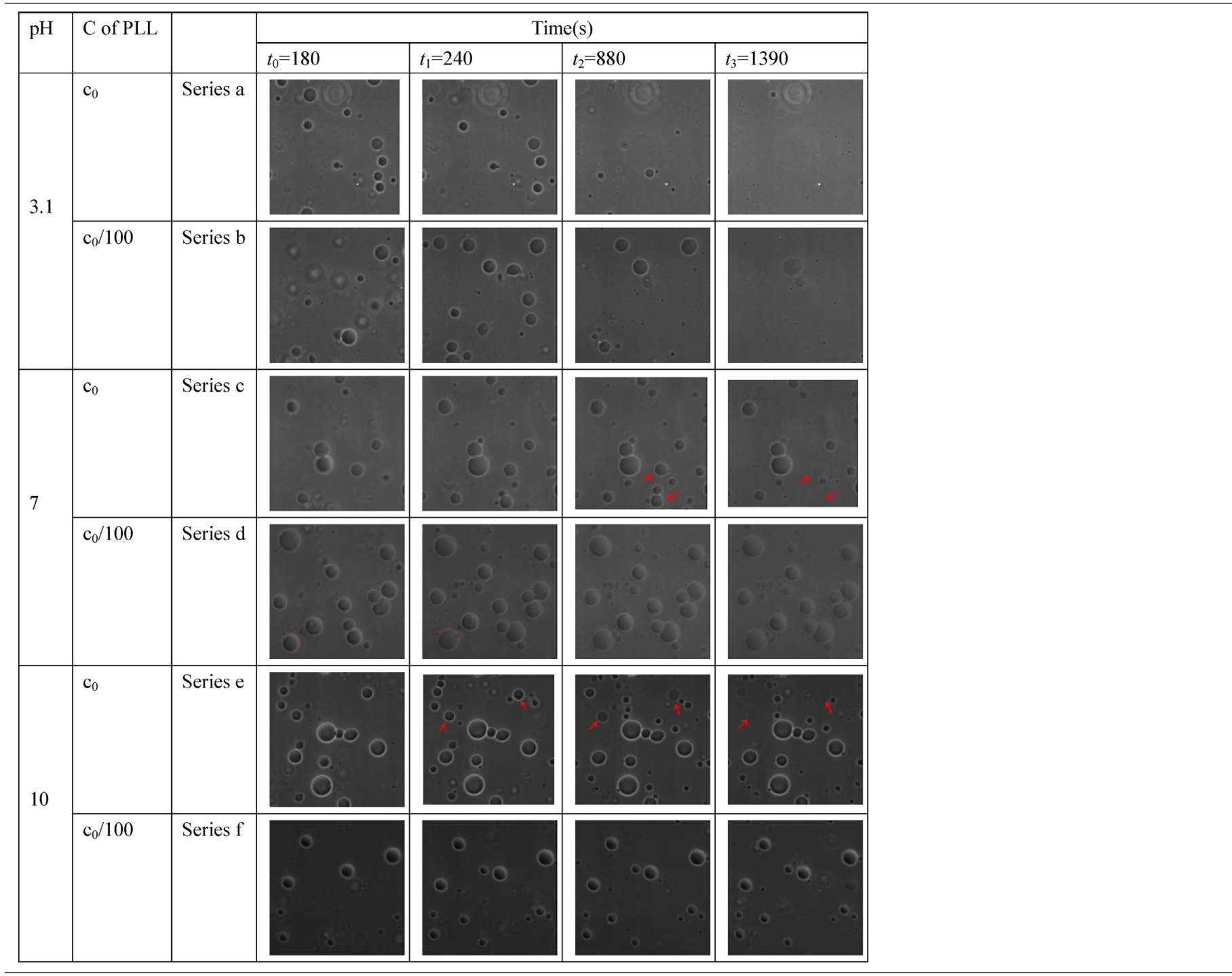

modulate the substrate/vesicle interaction and to induce three behaviors of reference for the vesicles: very strong interaction at low $\mathrm{pH}$ with immediate spreading of the vesicles on the surface followed by the rupture of their membrane, strong adhesion at moderate or high $\mathrm{pH}$ and high poly-L-lysine concentration, and weak adhesion at moderate or high $\mathrm{pH}$ and low poly-L-lysine concentration.

These three behaviors are first highlighted by direct observation using phase contrast microscopy under various conditions regarding poly-L-lysine concentration and $\mathrm{pH}$ of the vesicle suspension: membrane rupture, strong adhesion and weak adhesion. Then, the TIRIE signals obtained by the vesicles in the same conditions of poly-L-lysine concentration and $\mathrm{pH}$ are studied, and the results show that the TIRIE biosensor can sensitively discriminate the three behaviors of reference.

\subsection{Direct observation of vesicle adhesion}

The initial time of observation is set as $t=0 \mathrm{~s}$, the vesicle suspension is gently introduced into the observation chamber and the vesicles slowly sediment until $t=240 \mathrm{~s}$. They are visualized while being settled on the substrate. At $\mathrm{pH}=3$, the membrane of most vesicles break within $10 \mathrm{~min}$ after sedimentation and the vesicles totally disappear. The remaining vesicles break during 5 min in the gentle rinsing stage, regardless the $c_{0}$ or $c_{0} / 100$ substrate. Only very small ones (diameter $\approx 1 \mu \mathrm{m}$ ) do not break. This behavior discloses a very strong attractive interaction between the vesicles and the substrate, which leads to an increase of their membrane tension during their spreading on the surface and ends in membrane lysis. Under $\mathrm{pH} 7$ and 10 and with the $c_{0}$ surface, most vesicles do not break at the contact with the substrate. However, the sudden breakage of some vesicles is observed during the rinsing stage (see arrows in Table 1), which reveals that vesicles are strongly adhered on the surface. A small force induced by a shear flow is sufficient to increase the membrane tension, leading to its lysis. However, most vesicles are intact, with a good light contrast with the suspending solution. This shows that the membrane has been preserved and no large pores have been opened. Vesicles in contact with $c_{0} / 100$ are even more stable. They do not break within $20 \mathrm{~min}$ after introduction into the observation chamber, even when being 
gently rinsed. One can notice that some additional vesicles appear with time due to the long process of sedimentation throughout the whole microscopic chamber. Moreover, these vesicles are not removed by the small shear flow occurring during the very gentle final rinsing, which shows that the vesicles are not just settled on the surface but really adhere to it. This is the weak adhesion state.

\subsection{TIRIE biosensor results}

Experiments similar to the previous ones have been performed using the TIRIE biosensor with identically treated substrates and the same vesicle solutions. Typical signals obtained by the TIRIE biosensor are shown in Fig. 3(a)-(c) for the two substrates of $c_{0}$ and $c_{0} / 100$ and for three $\mathrm{pH}$ conditions, 3.1, 7 and 10. A detailed analysis of the signal unambiguously discriminates the different vesicle behaviors. Under $\mathrm{pH}=3$, the fast increase of the intensity of the signal is moderate and the further slow increase during sedimentation is very limited. The intensity of the signal decreases as rinsing proceeds. And it returns to its initial value before introduction of vesicles. The process shows that no more vesicles are present on the surface. This change of behavior is interpreted as follows. The first fast increase of the intensity of the signal is associated with the flow of vesicles that arrive and break on the treated surface. The signal is mainly due to the presence of lipids fragments in the suspension that result from the membrane breakage. When the flow is stopped, the vesicles, which are still in solution, start to sediment and break on the surface. The process leads to a slow and very limited increase of the signal intensity before their stabilization (when stabilized, all vesicles are broken). The lipid fragments are washed away during the rinsing stage and the signal returns to its initial value.

Under $\mathrm{pH} 7$ and 10 the increase of the signal intensity during stage 6 is very strong. It is due to intact vesicles that progressively adhere on the substrate and to lipid fragments resulting from those vesicles that break upon strong adhesion or are destroyed during their travel in the micro-fluidic channels of the TIRIE biosensor. The increase of the signal intensity observed when the flow rate is stopped is due to both the sedimentation of vesicles still in solution at the end of the introduction stage and the change of vesicle shapes that occurs when adhesion is strong. The maximum signal intensity observed for $c_{0} / 100$ is always more intense than that for $c_{0}$. The maximum value $\left(2.165 \times 10^{4}\right.$ grayscale $)$ is observed for $\mathrm{pH}=10$. This can be understood by considering that more vesicles remain intact on $c_{0} / 100$ than on $c_{0}$ and the contribution to the signal of an intact adhered vesicle is stronger than that due to the same quantity of lipids aggregated in small fragments. During the final washing stage, the intensity of the signal decreases (the lipid fragments of broken vesicles are washed away) but does not return to its initial value. The final signal is directly related to the amount of adhered vesicles. The most intense final signal is observed for $c_{0} / 100$ at $\mathrm{pH}=10$, in agreement with microscopic observations.

The effect of $\mathrm{pH}$ on vesicle adhesion is more specifically illustrated in Fig. 3(d) on $c_{0} / 100$, based on 3 experiments performed on a vesicle suspension where the $\mathrm{pH}$ has been adjusted to 3.1, 7 and 10 , respectively. One clearly sees that the maximum intensity of the signal increases with the $\mathrm{pH}$, due to a larger concentration of intact adhered vesicles. During the last rinsing stage, strongly adhered vesicles remain on the surface in the channel, and only small lipid fragments are removed. Logically, the signal observed at high $\mathrm{pH}$ does not change whereas the signal observed at the lowest $\mathrm{pH}$ decreases drastically and returns to its initial value, showing that no more on the surface.

A high value of the maximum signal intensity $\left(I_{\max }\right)$ indicates the presence of intact vesicles. The final $\left(I_{\text {final }}\right)$ signal represents intact adhered vesicles and the difference between maximum $\left(I_{\max }\right)$ and final $\left(I_{\text {final }}\right)$ signal intensities is the contribution to the signal of the

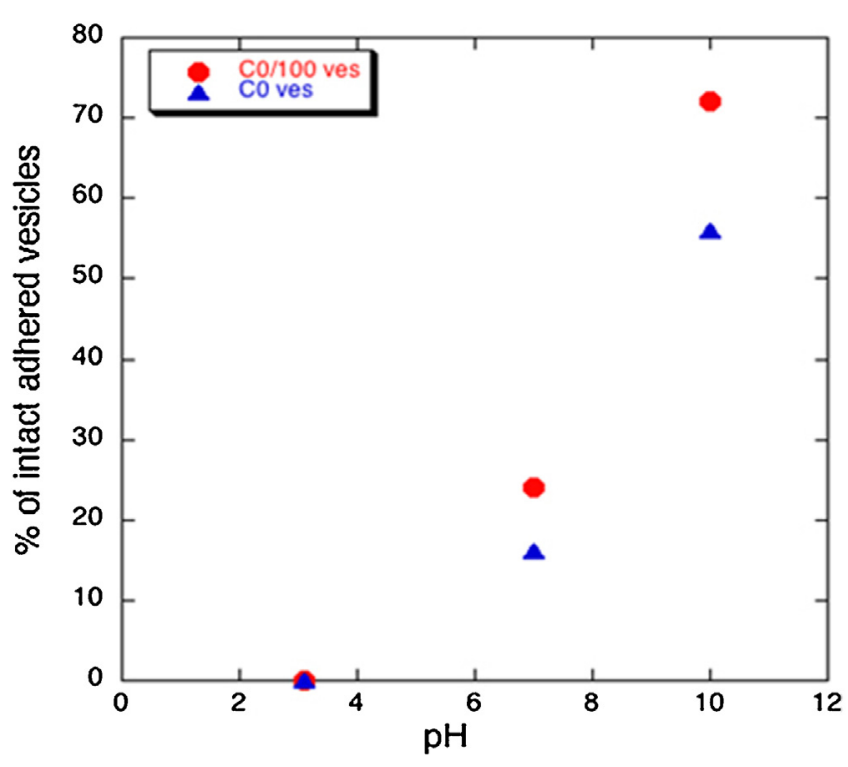

Fig. 4. Variation of the percentage of intact adhered vesicles versus the $\mathrm{pH}$.

lipid fragments deposited on the substrate originated from vesicles that have broken. Let's call $I_{\text {fragments, }}$, the total intensity of the signal when all vesicles are destroyed (maximum intensity for $\mathrm{pH}=3$ ) before removal by washing. As the signal of the TIRIE biosensor varies linearly with the amount of lipid fragments scattered on the surface, the ratio $\left(I_{\max }-I_{\text {final }}\right) / I_{\text {fragments }}$ represents the fraction of broken vesicles. The variation of the fraction of intact adhered vesicles, $1-\left(I_{\text {max }}-I_{\text {final }}\right) / I_{\text {fragments }}$ is plotted in Fig. 4 versus the $\mathrm{pH}$ and the nature of the surface, which demonstrates that TIRIE biosensorobservations and analysis are confirmed by phase microscopy observations. However, TIRIE biosensor has the advantage of dealing with a statistical assembly of vesicles. For instance it clearly

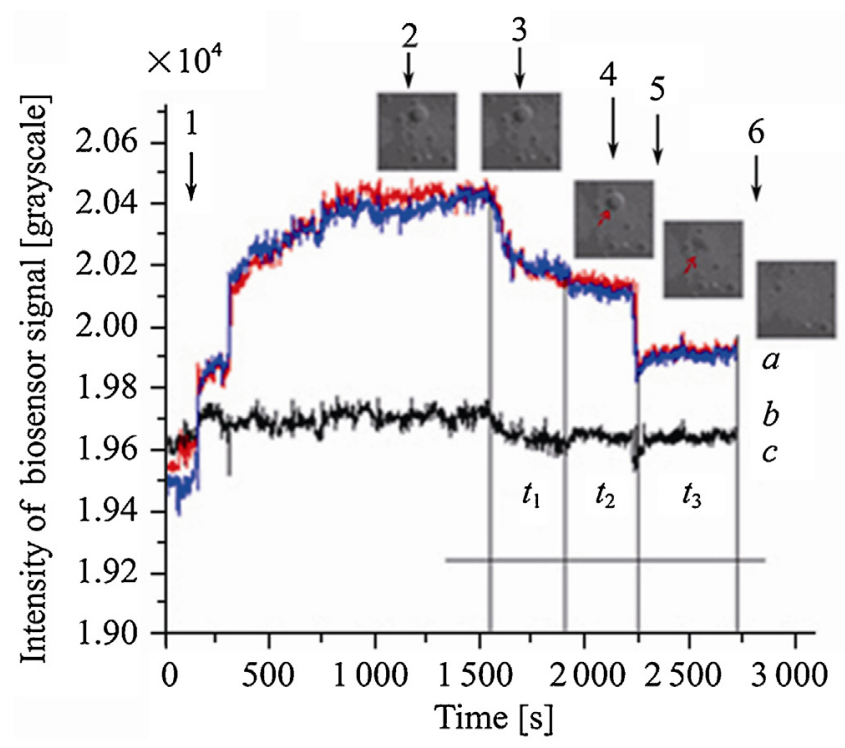

Fig. 5. Effect of the shear flow on the de-adhesion process of vesicles at $\mathrm{pH} 10$ and a poly-L-lysine modified gold surface with concentration of $c_{0} / 100$ for two similar experiments curve $a$ and $b$. The curve $c$ is a reference (no vesicle injection). Step 1-Calibration of the TIRIE signal; step 2-adhesion process; step 3-rinsing by glucose solution with flow rate $5 \mu \mathrm{L} / \mathrm{min}$ for $5 \mathrm{~min}$ ( $t_{1}$ interval); step 4 -flow rate $10 \mu \mathrm{L} / \mathrm{min}$ for $5 \mathrm{~min}$ ( $t_{2}$ interval); step 5 -flow rate $30 \mu \mathrm{L} / \mathrm{min}$ and step 6 -flow rate $30 \mu \mathrm{L} / \mathrm{min}$ (total duration $8 \mathrm{~min}$, interval $t_{3}$ ). The photos represent a spatial region where a vesicle, which is first adhered on the surface, is unbound and washed away by the flow. 
shows that the $c_{0} / 100$ surface is less attractive than the $c_{0}$ one even at $\mathrm{pH}=10$, but induces a real adhesion state since only a very small amount of material is washed away during the final rinsing stage.

Finally, in order to investigate the strength of vesicle adhesion and the de-adhesion processes, the flow rate was varied during the final rinsing stage. We use three successive rinsing flow rates: 5, 10 and $30 \mu \mathrm{L} / \mathrm{min}$. Experiments are performed under $\mathrm{pH}=10$ and on the $c_{0} / 100$ surface. The real-time process of vesicles adhesion and de-adhesion is shown in Fig. 5. The rinsing flow rate is progressively increased. The same experiment is reproduced in a small chamber using the microscope where a typical adhered vesicle is observed. During the first rinsing stage at $5 \mu \mathrm{L} / \mathrm{min}$ only a small fraction of material is removed (lipid fragments or a very small population of non-adhered vesicles), even if the flow rate increase to $10 \mu \mathrm{L} / \mathrm{min}$. It is necessary to increase the flow rate to $30 \mu \mathrm{L} / \mathrm{min}$ to detach the most strongly adhered vesicles. The two experiments are remarkably reproducible. At the end of rinsing, the intensity of the signal is similar to the initial signal before vesicle introduction: all vesicles have been unbound and removed by the flow.

\section{Conclusion}

In this article, GUVs were used to study the TIRIE biosensor response to various cases of strong interaction, strong and weak adhesion and de-adhesion of micron-size vesicles on a surface treated to modulate the vesicle-surface interaction. Adjustment of the $\mathrm{pH}$ in the vesicle suspension enabled to modulate vesicle-substrate interactions. The maximum signal intensity of the biosensor is relevant to determine the integrity of GUVs and the difference between maximum and final signal intensity obtained after gentle rinsing is an accurate measurement of the fraction of intact objects adhering on the surface. The TIRIE biosensor is suitable to determine the flow-rate threshold of vesicle unbinding from the substrate. We believe that the results obtained on GUVs will be valid for sensing other systems like living cells, solid particles or capsules used for drug delivery or by cosmetics industry.

\section{References}

[1] N.F. Pasco, R.J. Weld, et al., Development and applications of whole cell biosensors for ecotoxicity testing, Anal. Bioanal. Chem. 400 (2011) 931-945.

[2] Q. Yu, Y. Li, et al., An efficient design strategy for a whole-cell biosensor based on engineeredribosomebinding sequences, Anal. Bioanal. Chem. 401 (2011) 2891-2898.

[3] P.S. Dittrich, P. Schwille, An integrated microfluidic system for reaction highsensitivity detection, and sorting of fluorescent cells and particles, Anal. Chem. 75 (2003) 5767-5774

[4] J. Wegener, C.R. Keese, et al., Electric cell-substrate impedance sensing (ECIS) as a noninvasive means to monitor the kinetics of cell spreading to artificial surfaces, Exp. Cell Res. 259 (2000) 158-166.

[5] I. Giaever, C.R. Keese, Monitoring fibroblast behavior in tissue-culture with an applied electric-field, Proc. Natl. Acad. Sci. U.S.A. 81 (1984) 3761-3764.

[6] Y. Fang, Label-Free, Non-invasive biosensor cellular assays for cell adhesion, J. Adhes. Sci. Technol. 24 (10) (2010) 1011-1021.

[7] K.F. Giebel, C. Bechinger, et al., Imaging of cell/substrate contacts of living cells with surface plasmon resonance microscopy, J. Biophys. 76 (1999) 509-516.

[8] Y. Fang, A.M. Ferrie, et al., Resonant waveguide grating biosensor for living cell sensing, Biophys. J. 91 (2006) 1920-1945.

[9] J.J. Ramsden, S.Y. Li, et al., Kinetics of adhesion and spreading of animal cells, Biotechnol. Bioeng. 43 (1994) 939-945

[10] G. Jin, R. Jansson, H. Arwin, Imaging ellipsometry revisited: development for visualization of thin transparent layers on silicon substrates, Rev. Sci. Instrum. 67 (1996) 2930-2936.

[11] G. Jin, Y.H. Meng, et al., Development of biosensor based on imaging ellipsometry and nd biomedical applications, Thin Solid Films 519 (2011) 2750-2757.

[12] A.W. Szmodis, C.D. Blanchette, et al., Direct visualization of phase transition dynamics in binary supported phospholipid bilayers using imaging ellipsometry, Soft Matter 4 (2008) 1161-1164.

[13] L. Asinovski, D. Beaglehole, et al., Imaging ellipsometry: quantitative analysis, Phys. Status Solidi A 205 (2008) 764-771.

[14] D. Ausserre, M.P. Valignat, Wide-field optical imaging of surface nanostructures, Nano Lett. 6 (2006) 1384-1388.
[15] A. Gunnarsson, M. Bally, et al., Time-resolved surface-enhanced ellipsometric contrast imaging for label-free analysis of biomolecular recognition reactions on glycolipid domains, Anal. Chem. 84 (2012) 6538-6654.

[16] G. Jin, P. Tengvall, et al., A biosensor concept based on imaging ellipsometry for visualization of biomolecular interactions, Anal. Biochem. 232 (1995) 69-72.

[17] G. Jin, Y. Niu, Approach to quantitative detection of CD146 with the biosensor based on imaging ellipsometry, in: 4th European Conference of the International Federation for Medical and Biological Engineering (ECIFMBE 2008), 23-27 November 2008 Antwerp, Belgium, Springer, Berlin, Heidelberg, 2008, pp. 1046-1049.

[18] R. Lipowsky, The morphology of lipid-membranes, Curr. Opin. Struct. Biol. 5 (1995) 531-540.

[19] A. Boulbitch, Z. Guttenberg, et al., Kinetics of membrane adhesion mediated by ligand-receptor interaction studied with a biomimetic system, Biophys. J. 81 (2001) 2743-2751.

[20] R. Esquembre, S.N. Pinto, et al., Immobilization and characterization of giant unilamellar vesicles (GUVs) within porous silica glasses, Soft Matter 8 (2012) 408-417.

[21] M. Le Berre, A. Yamada, et al., Electroformation of giant phospholipid vesicles on a silicon substrate: advantages of controllable surface properties, Langmuir 24 (2008) 2643-2649.

[22] B.L.S. Mui, P.R. Cullis, et al., Osmotic properties of large unilamellar vesicles prepared by extrusion, Biophys. J. 64 (1993) 443-453.

[23] J. Nardi, T. Feder, et al., Electrostatic adhesion between fluid membranes: phase separation and blistering, Europhys. Lett. 37 (1997) 371-376.

[24] M. Abkarian, C. Lartigue, et al., Motion of phospholipidic vesicles along an inclined plane: sliding and rolling, Phys. Rev. E 63 (2001) 041906.

[25] T.J. Politano, V.E. Froude, et al., AC-electric field dependent electroformation of giant lipid vesicles, Colloids Surf. B 79 (2010) 75-82.

[26] L. Liu, Y.Y. Chen, et al., Improvement for sensitivity of biosensor with tota internal reflection imaging ellipsometry (TIRIE), Thin Solid Films 519 (2011) 2758-2762.

[27] A. Bandekar, S. Sofou, Floret-shaped solid domains on giant fluid lipid vesicles induced by pH, Langmuir 28 (2012) 4113-4122.

\section{Biographies}

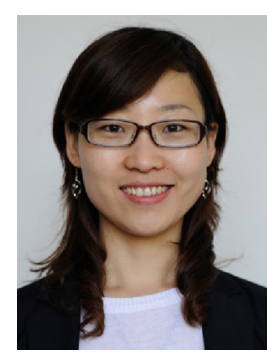

Li Liu received her PhD degree in Physics from Institute of Mechanics. Her research field is on the key techniques for biosensor based on Total reflection imaging ellipsometry.

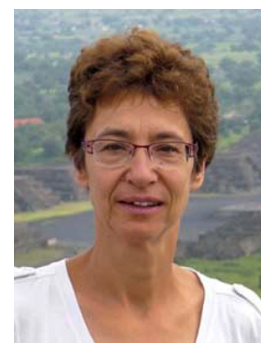

Annie Viallat is ingénieur of Ecole Polytechnique (Paris France). She received her PhD in Physics from the University of Grenoble (France) in 1987, working on PDMS/silica mixture and NMR. After a theoretical postdoctoral work on conjugated polymers in Materials Departments (UC Santa Barbara), she joined the Spectrométrie Physique lab (Grenoble) in 1989, studying polymer gels and heterogeneous polymer solutions. Her research moved to biological physics and biomechanics in 1999. Since 2005 , she is a group leader in the 'Adhesion and Inflammation' lab in Marseilles, interested in cell rheology and dynamics in biomimicking microflows of lipid vesicles, red and white blood cells. Her recent work also focuses on encapsulation of biological materials in lipid vesicles and the mucociliary clearance in airways.

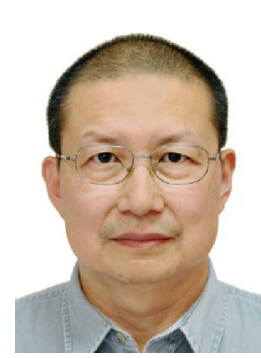

Gang Jin enrolled in ESPCI Paris, France, on the supervision of Professor A.C. Boccara, and received Doctoral Degree in Pierre \& Marie Curie University of Paris (Paris 6) during 1989-1993; From 1992-1994 he is an associate researcher at CNRS, Bellevue, Paris; He is the visiting scholar at Sweden Linkoping University in 1994-1996 for imaging ellipsometry development and its applications; Since 1996, he has served as a research professor at the Institute of Mechanics, Chinese Academy of Sciences, and engaged in systematic studies and development on label free detection protein microarray technique based on imaging ellipsometry, and succeeded in developing laboratory prototypes. In 2006, "the biosensors based on imaging ellipsometry" passed the achievement authentication organized by Chinese Academy of Sciences. With the concept of protein microarray biosensor based on imaging ellipsometry, he solved problems in working principles, methodology, build-up of experiment system and biomedical applications, and finally developed a practical label free protein microarray biosensor. 